\title{
GIMNASIA DE COMPETICIÓN EN LA TRAYECTORIA ESCOLAR DE ESTUDIANTES INGRESANTES A LA LICENCIATURA EN EDUCACIÓN FÍSICA EN PARANÁ - BRASIL ${ }^{1}$
}

\author{
GINÁSTICAS COMPETITIVAS NO PERCURSO ESCOLAR DE INGRESSANTES EM \\ CURSOS DE LICENCIATURA EM EDUCAÇÃO FÍSICA DO PARANÁ - BRASIL
}

\begin{abstract}
COMPETITIVE GYMNASTICS AT THE SCHOLAR HISTORY OF NOVICE STUDENTS OF PHYSICAL EDUCATION TEACHER EDUCATION DEGREE IN PARANÁ - BRAZIL
\end{abstract}

\author{
Bruna Sontag ${ }^{3}$, Verónica Gabriela Silva Piovani ${ }^{4}$, Ademir Faria Pires ${ }^{5}$, Eliane Josefa \\ Barbosa Dos Reis ${ }^{6}$, leda Parra Barbosa Rinaldi ${ }^{3}$ \\ sontagbruna@gmail.com; veropiovani@hotmail.com; afariapires@gmail.com; \\ elianejbr13@gmail.com; parrarinaldi@hotmail.com
}

${ }^{3}$ Universidad Estatal de Maringá, Brasil ${ }^{4}$ Universidad Estatal del Oeste de Paraná, Brasil ${ }^{5}$ Universidad Paranaense, Brasil ${ }^{6}$ Universidad Estatal de Paraná, Brasil

Envío Original: 2019-05-20 Reenviado: 2019-09-05 Aceptado: 2019-11-01

Publicado en versión en español: 2020-06-04

Doi: https://doi.org/10.15517/pensarmov.v18i1.42148

\begin{abstract}
Resumen
El objetivo fue analizar las experiencias vivenciadas por los estudiantes que ingresaron a cursos de Licenciatura en Educación Física en el Estado de Paraná (Brasil) con la gimnasia de competición durante la Educación Primaria y Secundaria. La investigación se caracterizó como descriptiva con abordaje cualitativo y cuantitativo. Participaron 168 estudiantes que ingresaron en el año 2017 en cursos de Licenciatura en Educación Física de cuatro universidades públicas de Paraná. Fue utilizado un cuestionario con información sociodemográfica, preguntas abiertas y cerradas sobre la experiencia del contenido de gimnasia de competición en el contexto escolar. Para analizar las preguntas abiertas fue utilizado el análisis de contenido. En el análisis de información sociodemográfica y preguntas
\end{abstract}

\footnotetext{
${ }^{1}$ Versión traducida al español. Original en portugués disponible en: Sontag, B., Silva Piovani, V., Faria Pires, A., Barbosa dos Reis, E., e Parra Barbosa Rinaldil. (2020). Ginásticas competitivas no percurso escolar de ingressantes em cursos de licenciatura em educação física do Paraná - Brasil. Pensar En Movimiento: Revista de Ciencias del Ejercicio y la Salud, $18(1)$, e37302. doi: https://doi.org/10.15517/pensarmov.v18i1.37302

${ }^{2}$ El estudio conto con el financiamento del Conselho Nacional de Desenvolvimento Científico e Tecnológico (CNPq) - Programa Institucional de Bolsas de Iniciação Científica (PIBIC).
} 
cerradas se usó estadística descriptiva. Se evidenció escasa experiencia por parte de los estudiantes con las modalidades de gimnasia de competición y falta de oportunidad de estos alumnos de vivenciar y reflexionar sobre la gimnasia en la Educación Física escolar.

Palabras-clave: educación física escolar, licenciatura, formación profesional, gimnasia.

\title{
Resumo
}

O objetivo foi analisar as experiências vivenciadas pelos acadêmicos ingressantes de cursos de Licenciatura em Educação Física do Estado o Paraná com a ginástica de competição na Educação Básica. A pesquisa caracterizou-se como descritiva, com abordagem qualitativa e quantitativa. Participaram 168 estudantes ingressantes no ano de 2017 no curso de Educação Física Licenciatura de quatro universidades públicas do Paraná. Foi utilizado um questionário com informações sócio demográficas e questões abertas e fechadas sobre a experiência do conteúdo 'ginásticas de competição no contexto escolar'. Foi utilizada a análise de conteúdo para as questões abertas e a estatística descritiva para a análise das informações sócio demográficas e questões fechadas. Evidenciou-se escassa experiência por parte dos estudantes com as modalidades de ginástica de competição e a falta de oportunidade destes alunos de vivenciar e refletir sobre a ginástica na Educação Física escolar.

Palavras-chave: educação física escolar, licenciatura, formação profissional, ginástica.

\begin{abstract}
The aim was to analyze the experiences lived by novice students of Physical Education Teacher Education graduation in Paraná State (Brazil) with the competitive gymnastics in the Primary and Secondary Education. The research was descriptive and has qualitative and quantitative approach. Participated 168 students who enter to the Physical Education Teacher Education course in four public universities of Paraná in 2017. Was used a questionnaire with socio demographic information, open and close questions about the experience of the competitive gymnastics content at school. Open questions were analyzed by content analysis. Socio demographic and close questions were analyzed by descriptive statistic. It was evidenced the students had little experience with the competitive gymnastics modalities and they had lack of opportunities of experience and reflect about gymnastic in the Scholar Physical Education.
\end{abstract}


Key words: scholar physical education, physical education teacher education, professional formation, gymnastic.

\section{INTRODUCCIÓN}

Al ingresar en un curso de formación profesional para la docencia, el futuro profesional trae consigo un repertorio de aprendizajes adquiridos por medio de observaciones de profesores, y posee conceptos sobre lo que significa ser profesor a partir de experiencias personales vividas en su papel de alumno (Graça, 2014). Este proceso de socialización anticipatoria, que ocurre a partir de los intercambios entre individuo y sociedad, promueve aprendizajes y experiencias que capacitan a las personas para la vida activa, lo cual es primordial para la concepción de la profesión (Barros, 2011).

Dentro de esos aprendizajes anteriores a la formación inicial, se encuentra el aprendizaje de la gimnasia en la escuela, la cual tiene un papel relevante en la formación de los alumnos. Su vivencia en las lecciones de educación física (EF) muestra un gran potencial en el desarrollo de ricas experiencias y la construcción del conocimiento de los educandos (Andrade, Da Silva Rocha, Paraíso Alves y Vinciprova Fonseca, 2016; Ramirez Costa, de Carvalho Macías, Da Cunha Faro y da Silva Matos, 2016). Todavía dentro del contenido gimnástico se encuentran las distintas modalidades de gimnasia de competición, las cuales se legitiman como prácticas altamente ricas y positivas para el contexto escolar, ya que pueden ofrecer posibilidades de estimulación y exploración motriz, cognitiva, afectiva y social, además de proporcionar momentos de alegría y placer a los educandos a partir de una lección diferenciada y desafiante (Merida, Nista-Piccolo y Merida, 2008; Soares et al., 2009).

Sin embargo, distintos estudios evidencian la ausencia de la enseñanza de la gimnasia en la escuela. Dentro de los principales motivos señalados para esta situación, se destaca la capacitación profesional insuficiente para este contenido, así como la falta de equipamiento y materiales adecuados, la incoherencia entre los contenidos aprendidos en la formación inicial y la realidad escolar, la inseguridad de trabajar un contenido "peligroso" que podría amenazar la seguridad de los alumnos, y la visión de deporte competitivo que posee la gimnasia (Ayoub, 2007; Ramirez Costa et al., 2016; Porto, 2014; Schiavon y Nista-Piccolo, 2007).

Así, la presencia (o ausencia) de las modalidades de gimnasia de las asignaturas programadas en el período escolar se refleja en la formación académica y profesional de quienes en un futuro optarían por seguir una carrera docente en EF. El repertorio de las experiencias gimnásticas aprendidas en la vida escolar prepara a los futuros profesores para la adquisición de nuevos conocimientos en cada modalidad de la gimnasia, que aprenderán en el curso de formación inicial (Barbosa-Rinaldi y Machado de Souza, 2003). 
De este modo, al considerar que los alumnos que ingresan en los cursos de licenciatura tuvieron ya vivencias de la disciplina de la EF escolar durante su Educación Básica, surgen los siguientes cuestionamientos: ¿Cuáles fueron las modalidades de gimnasia competitiva que experimentaron los académicos del primero año del programa de grado de las universidades públicas del Estado de Paraná? ¿Cuál o cuáles fueron las estrategias de enseñanza vivenciadas por los estudiantes para el desarrollo de dicho contenido? ¿Cuál es la percepción que tienen ellos acerca de la influencia de sus experiencias sobre su vida y su futuro profesional? Por lo tanto, el objetivo del estudio fue analizar las experiencias vividas por los académicos que ingresan a los cursos de licenciatura en educación física del estado de Paraná, en relación con la gimnasia competitiva en su educación básica.

\section{METODOLOGÍA}

La investigación fue descriptiva con un abordaje cualitativo de los datos (Cervo y Bervian, 1996). La muestra fue de 168 estudiantes (84 mujeres y 84 hombres) del curso de licenciatura en EF, que ingresaron en el año 2017 en cuatro universidades públicas del estado de Paraná. La edad promedio de los participantes fue de 19,66 años (desviación estándar = 3,68 años). Se utilizó un cuestionario con información sociodemográfica y preguntas abiertas y cerradas sobre la enseñanza de la gimnasia competitiva en la educación básica e intermedia.

El cuestionario se construyó para efectos de esta investigación y pasó por una evaluación de contenido y claridad del lenguaje por parte de tres profesores de EF, especialistas en el área de gimnasia. En cada ítem, los especialistas indicaron si la pregunta era no válida, poco válida o válida; además, tuvieron la opción de indicar sus sugerencias o comentarios para la construcción del instrumento (Dos Santos y Gheller, 2012). Así, el cuestionario se ajustó conforme a las sugerencias de los profesores especialistas del área.

Para analizar los datos se utilizó el análisis de contenido (Bardin, 2011) de la información obtenida a partir de las preguntas abiertas y el análisis de estadística descriptiva con la información sociodemográfica. La investigación se inició posterior a la aprobación del Comité Permanente de Ética en Investigación con Seres Humanos de la Universidad Estatal de Maringá (Criterio N².410.354).

\section{PRESENTACIÓN Y DISCUSIÓN DE LOS RESULTADOS}

Al evaluar los datos sociodemográficos se observó que, de los 168 participantes del estudio, 50 pertenecían a la universidad \#1 (U1), 39 a la universidad \#2 (U2), 47 a la 
universidad \#3 (U3) y 32 a la universidad \#4 (U4). Todos los estudiantes estaban cursando el primer año de la carrera de licenciatura en EF: 57 en horario matutino, 45 por la noche y 66 en horario integral (curso con lecciones tanto en el período matutino como vespertino).

Al proceder a analizar el conocimiento que tenían los estudiantes sobre gimnasia competitiva, se observó que una gran parte de ellos la entienden como una competición propiamente dicha, en la cual se clasifican y premian los mejores (85 menciones).También la entienden como gimnasia artística/olímpica y rítmica (38 menciones) y como una modalidad deportiva de alto rendimiento y perfección técnica (33 menciones).

Solamente 31 estudiantes reportaron haber recibido gimnasia de competición en las lecciones de EF durante su educación básica. De estos, 21 manifestaron haber realizado contenidos de gimnasia artística, 15 recibieron gimnasia rítmica, 16 gimnasia aeróbica, uno recibió rueda alemana o Rhönrad y uno más, trampolín acrobático.

Se percibe que el conocimiento acerca de la gimnasia de competición de los estudiantes está de cierta forma influenciado por los medios de comunicación (Araújo, 2017) pues, entre las modalidades más citadas por los estudiantes están aquellas que poseen mayor divulgación en los medios: la gimnasia artística y la rítmica. Es imposible disociar el deporte de los medios; esa relación viene alterando progresivamente las percepciones y el modo en que se practica el deporte (Betti, 1997). No obstante, el significado de deporte que traen los medios no refleja su totalidad y eso acarrea prejuicios para el deporte educacional (Lisbôa \& Pires, 2016).

Entre aquellos estudiantes que tuvieron el contenido en sus clases de EF se observaron ocho estrategias metodológicas experimentadas: la demostración para una posterior ejecución de movimientos (19 menciones); la utilización de videos (18 menciones); la lectura de textos, libros e investigaciones en Internet (16 menciones); la creación de coreografías (12 menciones); la observación de presentaciones (9 menciones); trabajos en equipos o grupos pequeños con ayuda y corrección entre los mismos compañeros (9 menciones); la utilización de progresiones para la enseñanza de movimientos más complejos (7 menciones) y la utilización de circuitos con aparatos (3 menciones).

En las estrategias enumeradas se observa un cierto equilibrio entre experimentar los movimientos de forma activa y pensar sobre el movimiento. En un estudio realizado con alumnos de $8^{\circ}$ grado de enseñanza básica, en el cual se trabajaron los contenidos de gimnasia artística, acrobática y rítmica, se evidenció esa preocupación por englobar las tres dimensiones del contenido (procedimental, conceptual y actitudinal), lo cual estimula el pensamiento crítico del alumno, además de hacerlo reflexionar sobre la gimnasia (Maldonado y Bocchini, 2015). 
La gimnasia en la EF escolar debe considerarse un tema de cultura corporal, siendo trabajada, experimentada, problematizada, conocida y transformada (Marcassa, 2004). Y la experiencia es una buena forma de aprendizaje de la gimnasia, pues su práctica desarrolla aspectos cognitivos, motores y socioafectivos (Ramirez Costa et al., 2016).

Daolio (1996) propone que, al inicio de los primeros años de la EF escolar, los alumnos deben experimentar la mayor cantidad posible de gestos motores, entender sus significados y sus formas de ejecución. Luego, el alumno debe desarrollar y reconstruir las técnicas deportivas, no enfocándose en la técnica, sino en las relaciones con el espacio, con sus colegas o con los materiales. Finalmente, hacia el final del primer año y a lo largo del segundo, se deben ampliar los bjetivos de la EF, llevando a los alumnos a pensar hipotéticamente sobre la cultura corporal, comprendiéndola y criticándola.

Las consecuencias de la ausencia o presencia de vivencias en la gimnasia competitiva se analizaron conforme a su incidencia en la vida de cada estudiante y en su formación profesional (Tabla 1).

\section{Tabla 1.}

Consecuencias de la ausencia o presencia de vivencias de gimnasia de competición en la EF escolar, para la vida y la formación profesional de los estudiantes de la investigación.

\begin{tabular}{lc}
\hline $\begin{array}{l}\text { Indicadores temáticos de las consecuencias de la falta o } \\
\text { presencia de experiencias en gimnasia de competición en la EF } \\
\text { escolar }\end{array}$ & $\begin{array}{c}\text { \# de } \\
\text { menciones* }\end{array}$ \\
\hline $\begin{array}{l}\text { Consecuencias para la vida de los estudiantes } \\
\text { Falta de conocimiento sobre las características de la gimnasia } \\
\quad \text { y sus distintas modalidades. }\end{array}$ & 48 \\
$\begin{array}{l}\text { Falta de conocimiento corporal y motor. } \\
\text { El poco conocimiento ofrecido estimuló a los estudiantes que } \\
\text { recibieron el contenido a buscar saber más sobre el tema. }\end{array}$ & 15 \\
\hline $\begin{array}{l}\text { Consecuencias para la formación profesional en EF } \\
\text { La falta de conocimiento y experiencia práctica dificulta el } \\
\text { aprendizaje durante los estudios de la especialidad. }\end{array}$ & 11 \\
$\begin{array}{l}\text { No tener la vivencia del contenido por parte de los profesores } \\
\text { de EF escolar despierta nuevas actitudes para revertir eso } \\
\text { en el futuro como profesor de EF. }\end{array}$ & 04 \\
\hline $\begin{array}{l}\text { No tuvo consecuencias para la vida ni para el futuro profesional } \\
\text { en la EF }\end{array}$ & 16 \\
\hline $\begin{array}{l}\text { No respondieron pues no recibieron ese contenido en la EF } \\
\text { escolar }\end{array}$ & 25 \\
\hline
\end{tabular}

Nota: * Los indicadores temáticos pueden haber sido mencionados más de una vez, o ninguna vez por cada participante. Por eso, en este caso, hay menos menciones que el número total de individuos en la investigación. Fuente: elaboración propia 
Las consecuencias para la vida de los estudiantes con mayor número de citas fueron la falta de conocimiento sobre las características de la gimnasia y de sus distintas modalidades (48 menciones) y la falta de conocimiento corporal y motor (15 menciones). Se constató en este estudio, así como en el de Barbosa-Rinaldi y Machado de Souza (2003), que la gimnasia no se está aplicando como debería y que la mayoría de los alumnos nunca tuvieron la modalidad dentro de las lecciones de EF. Es importante enfatizar que la gimnasia es recomendada como un contenido que debe ser tratado en la EF escolar, tanto en los documentos normativos de la educación básica brasileña (Base Curricular Nacional Común) (Brasil, 2018), como en los paranaenses (Directrices Curriculares de la Educación Básica del Estado de Paraná para la Educación Física) (Secretaria de Estado da Educação do Paraná, 2008).

Por otra parte, el poco conocimiento ofrecido, estimuló a una minoría de los estudiantes (aquellos que tuvieron el contenido en las clases) a buscar más sobre el tema (7 menciones) (tabla 1). Aunque esa actitud de autonomía sea deseable en la enseñanza de la EF, es posible verificar que fue mencionada por una minoría del total de participantes de la investigación. La EF escolar debe incitar, mediante el conocimiento corporal popular, a un conocimiento organizado, crítico y autónomo sobre la cultura humana del movimiento (Daolio, 1996). Pero ese conocimiento autónomo debe ofrecerse a todos los alumnos por medio de una estrategia metodológica planificada.

En el caso específico de la gimnasia, su práctica en la escuela debería abrir nuevas posibilidades de experiencias corporales, para enriquecer la cultura corporal de niños y niñas y permitir el significado propio de los alumnos (Soares et al., 2009), pero no a partir de la escasez sino por la oferta de una vasta amplitud de experiencias.

Entre las consecuencias de la ausencia o presencia de las experiencias de la gimnasia de competición en la EF escolar para la formación profesional en EF, los estudiantes resaltan el perjuicio en relación con la experiencia para la práctica en las clases de formación universitaria (11 menciones). En el estudio de Ramirez Costa et al. (2016), de catorce profesores de EF entrevistados, once afirmaron no trabajar la gimnasia en sus lecciones, reafirmando el hecho de que el contenido no se trabaja como debiera. Esto porque, de acuerdo con los documentos normativos de la educación básica brasileña y paranaense, la gimnasia competitiva debe cubrirse en el ámbito escolar, haciendo un recorrido por sus características, elementos fundamentales y su historia, entre otros (Brasil, 2018; Secretaria de Estado da Educação do Paraná, 2008). También se describe que los cursos de formación universitaria no preparan a los futuros profesionales de manera adecuada, lo que se suma a la falta de experiencia existente, causada por la ausencia de la modalidad en las escuelas. 
La presencia de la gimnasia competitiva en las escuelas es importante para que los estudiantes adquieran un acervo o cúmulo de movimientos que los prepare para los cursos de formación universitaria en EF y para la práctica profesional futura (Machado de Souza, 1997). Además, las experiencias vividas durante la escuela son incorporadas y portadas por los académicos, interfiriendo en la escogencia de una carrera y en el accionar profesional futuro de estos académicos (Barbosa-Rinaldi y Machado de Souza, 2003).

Los alumnos también señalaron, como consecuencia de la falta o presencia de experiencias vivenciadas en la gimnasia de competición en la EF escolar, el despertar de nuevas actitudes como futuros profesores en el área, llevándolos a no omitir el abordaje del contenido gimnástico (4 menciones). Es decir, aprendieron lo que no deberían hacer, como futuros profesionales de EF. Esta actitud es de una minoría, pues exige una cierta madurez personal de parte de los estudiantes egresados de la educación básica. En términos generales, el futuro profesional no tuvo el contenido durante su vida escolar, muchas veces recibío la gimnasia de manera defectuosa durante sus estudios universitarios, y no se siente suficientemente seguro como para aplicar la modalidad en sus lecciones. Así, el profesor de EF acaba privando a sus alumnos del universo de la gimnasia (Barbosa-Rinaldi y Machado de Souza, 2003).

Un grupo de estudiantes no cree que la falta de este contenido en la Educación Básica les influya de algún modo. En este caso, se puede entender que el desconocimiento de las modalidades genera una divergencia con respecto a lo que las publicaciones abordan acerca de la importancia de la gimnasia en el desarrollo de los individuos. Esto porque, entre los beneficios del aprendizaje de la gimnasia, sea o no competitiva, se citan el desarrollo de las acciones motrices, el trabajo de aspectos afectivos y cognitivos, y la prevención de las enfermedades causadas por el sedentarismo (Andrade et al., 2016; Ramirez Costa et al., 2016).

Por otra parte, en el análisis sobre las posibles contribuciones del desarrollo del contenido de gimnasia competitiva en las clases de EF escolar percibidas por los estudiantes, se hicieron evidentes dos temáticas: las posibles contribuciones para el aprendizaje y desarrollo de los individuos, y las posibles contribuciones para la docencia en EF (tabla 2). 


\section{Tabla 2.}

Posibles contribuciones del desarrollo del contenido de gimnasia competitiva en las lecciones de EF escolar, conforme a los participantes de la investigación.

\section{Indicadores temáticos de}

$\mathrm{N}^{0}$ de

menciones*

\section{Posibles contribuciones para el aprendizaje y desarrollo de los individuos}

Contribuye al conocimiento de saberes sobre la cultura corporal del movimiento (más allá de los deportes tradicionalmente abordados en la EF escolar).

Contribuye al desarrollo de capacidades físicas, corporalidad, habilidades y capacidades que otros deportes no desarrollan.

Contribuye al desarrollo o aprendizaje cognitivo, social y afectivo de las personas.

Contribuye a aprender a enfrentar situaciones de disputa o competencia.

\section{Posibles contribuciones a la docencia en EF}

Contribuye a que el docente de EF sepa detectar quién puede tener las condiciones y la voluntad de convertirse en atleta de la modalidad (y pueda buscar esa oportunidad fuera de la escuela).

Contribuye a que el docente, a través de la EF escolar, estimule la práctica de actividad física para la vida o el ocio (ofrecer la oportunidad para quien no tiene los medios de conocerla fuera de la escuela).

Mejora o amplía el rango de conocimiento base de los estudiantes que desean seguir su formación profesional en el área de la EF.

Contribuye a que los futuros profesores de EF sepan aplicar nuevas metodologías en las lecciones e incluyan a los alumnos que no participan de las clases a partir de la oferta de otros contenidos.

\section{No ofrece contribuciones}

No contribuye porque en la educación física escolar la competición no es el objetivo.

No contribuye porque hay una falta de equipamiento para su práctica en las escuelas.

No contribuye porque no es interesante para los alumnos

Nota: * Los indicadores temáticos pueden haber sido mencionados más de una vez, o ninguna, por cada participante. Por ello, en este caso, hay más menciones que número de individuos participantes. Fuente: elaboración propia. 
Los estudiantes definen que la gimnasia competitiva en el período escolar es importante para el desarrollo de los individuos porque contribuye a la obtención de conocimiento sobre la cultura corporal del movimiento, más allá de los deportes tradicionales (68 menciones). Más aún, manifiestan su contribución para el desarrollo de habilidades corporales de manera distinta a otras prácticas corporales (25 menciones). En ese sentido, el contenido de gimnasia es muy rico y proporciona a los alumnos oportunidades y estímulos motores, creativos sociales, de cooperación y ritmicidad de manera tan singular y diferenciada, que se legitima su importancia. Es un medio para que el alumno conozca las posibilidades de su cuerpo y desarrolle aspectos cognitivos, sociales y físicos (Andrade et al., $\underline{2016})$.

Otro indicador descrito por los estudiantes fue que la gimnasia de competición contribuye al desarrollo cognitivo, social y afectivo (21 menciones). La gimnasia engloba objetivos como el trabajo de lo cognitivo, de lo social, de lo afectivo, de lo motor, de los alumnos, abriendo oportunidades para vivencias que, fuera de las lecciones de EF, para muchos serían imposibles. Pero la gimnasia va más allá: contribuye a la formación humana, proporcionando a los alumnos la cooperación, la confianza, la autonomía y el placer de practicar actividad física o deportes (Barbosa-Rinaldi y Cesário, 2010; Merida et al. 2008; Melo Oliveira y de Oliveira Porpino, 2010).

Hubo estudiantes que se refirieron a la contribución de este contenido para prepararse para situaciones competitivas (5 menciones). Sin embargo, se debe enfatizar que la gimnasia de competición en las escuelas no debe circunscribirse solamente a la técnica o a perseguir el rendimiento. En ese sentido, Cavalcanti (2007), al referirse a la gimnasia rítmica, afirma que a pesar de que esa modalidad, algunas veces se ve únicamente como de alto rendimiento, su contenido es muy amplio, abarcando la danza, el circo, la gimnasia propiamente dicha y el deporte, lo cual es enriquecedor para el alumno.

No se trata de negar el deporte escolar, sino de insertarlo en un contexto que responda a la formación humana, ya que las contribuciones que el deporte escolar puede aportarle al alumno son diversas, como la capacidad crítica y la autonomía (Melo Oliveira y de Oliveira Porpino, 2010). Las modalidades competitivas "deben ser contenidos de la educación física escolar, mas no deben ser un fin en sí mismas" (Barbosa-Rinaldi y Machado de Souza, 2003, p.166). En ese sentido, Andrade et al. (2016) describen que la gimnasia artística (una modalidad de gimnasia competitiva) debe ser vista dentro del currículo de la EF escolar como una actividad física de base, un elemento formativo que es parte de la cultura corporal y no como un deporte de alto rendimiento.

Entre las "posibles contribuciones para la docencia en EF", 18 estudiantes respondieron que el trabajo del contenido de la gimnasia competitiva en las lecciones de EF 
le posibilita al docente encontrar nuevos interesados en darle continuidad a la modalidad, es decir, nuevos atletas. Se debe resaltar que la formación de atletas y el entrenamiento de alto rendimiento no es ni debe ser el objetivo de la gimnasia escolar. Al mismo tiempo, este contenido debe ser tematizado en las escuelas, pues es en este contexto que se ponen a disposición las oportunidades para que los alumnos conozcan y vivan nuevas experiencias y, en caso de que así lo deseen, orientarse hacia una especialización (Barbosa-Rinaldi y Machado de Souza, 2003).

También se observó una posible contribución para que el docente, a través de la EF escolar, estimule la práctica de actividad física para la vida y el ocio (16 menciones), ya que para muchos estudiantes el único contacto con el deporte es durante las lecciones de EF escolar. Se entiende como relevante el trabajo de las modalidades de gimnasia competitiva, para la búsqueda de la salud y la calidad de vida, para aprovechar el placer de practicar algo, de sentirse aceptado y capaz, teniendo experiencias nuevas que contribuyan para la formación motriz, cognitiva, afectiva y social de los estudiantes (Andrade et al. 2016; BarbosaRinaldi y Cesário, 2010; Ramirez Costa et al. 2016; Merida, et al. 2008; Melo Oliveira y de Oliveira Porpino, 2010).

Hubo estudiantes que entendieron la importancia de la gimnasia competitiva en el período escolar para ampliar la gama de aprendizajes de futuros profesionales en el área de la EF (6 menciones). Esto es relevante en la medida en que es necesario romper con el ciclo de la ausencia del contenido en las escuelas. Al trabajarse adecuadamente en la vida escolar, la gimnasia será parte de la formación educativa de niños y niñas y, en el caso de que decidieran optar por estudiar para profesionales en EF, facilitará y enriquecerá la fase académica, proporcionando mayores posibilidades de una práctica profesional futura de buena calidad (Barbosa-Rinaldi y Machado de Souza, 2003; Schiavon y Nista-Piccolo, 2007).

También se evidenció la idea de que el contenido de gimnasia de competición colabora para la inclusión de los alumnos que no participan de las lecciones y para la aplicación de nuevas metodologías (5 menciones). De hecho, en las Directrices Curriculares Estatales para la EF en Paraná, se defiende que el trabajo con estas modalidades debe permitir la participación de todos los estudiantes, respetando sus limitaciones e individualidades (Secretaria de Estado da Educação do Paraná, 2008).

Por el contrario, algunos estudiantes no percibieron que existieran contribuciones de la enseñanza del contenido de gimnasia competitiva en la EF, alegando que en la EF escolar la competición no es el objetivo (3 menciones), que la falta de equipamiento para la práctica en las escuelas la vuelve carente de importancia (2 menciones) y que los estudiantes en las escuelas no encuentran el contenido interesante (2 menciones). 
La falta de materiales y espacio para la práctica de la gimnasia en las escuelas es una realidad en todo el país. En un estudio realizado con catorce profesores de escuelas municipales de Belém-Pará, se verificó que la falta de materiales y espacio en las escuelas muchas veces perjudican la seguridad de los alumnos. Ese es uno de los motivos por los cuales la gimnasia termina por dejarse a un lado. En este mismo estudio, se observó que las mayores dificultades entrentadas para trabajar el contenido gimnástico, principalmente con adolescentes, fueron la falta de respeto e interés de los alumnos, aliados con el sedentarismo (Ramirez Costa et al., 2016).

Mientras tanto, Betti (1999) afirma que, aliado a las condiciones precarias para el trabajo de actividades físicas en las escuelas, también hay profesores que no demuestran interés en conocer más sobre las modalidades de gimnasia. Además alegan no estar habilitados para enseñar esos deportes, debido a cierta carencia en su formación académica.

\section{CONSIDERACIONES FINALES}

La evidencia muestra la falta de oportunidad que tuvieron los estudiantes investigados de tener vivencias y reflexionar sobre la gimnasia en general y de las modalidades competitivas en particular. De esta manera, se percibe una cierta omisión de la EF escolar a la hora de llevar adelante su objetivo de enseñar y procurar que cada estudiante construya su conocimiento sobre la cultura corporal de movimiento.

Se percibe que las informaciones e influencias provenientes de los medios de comunicación, en especial en la televisión—que es la más común entre los brasileños—se integran a los saberes del alumno. Así, los medios de comunicación tienen un papel importante en la formación de significados sobre el deporte en jóvenes y niños. Esto no sería diferente con la gimnasia de competición, en el cual mucho del conocimiento de la mayoría de los estudiantes sobre las modalidades provienen de esos medios de comunicación y no se amplía con la EF escolar.

Las modalidades de gimnasia de competición que los estudiantes experimentaron en la EF escolar fueron, en muchos casos, ninguna, y la minoría que tuvo el contenido, vivenció las modalidades más presentes en los medios: gimnasia artística y rítmica. $Y$ la gimnasia aeróbica, por estar ligada al contacto con los gimnasios. De esta forma, en la EF escolar el alumno no trasciende el conocimiento de su día a día y, en los pocos casos en que recibe el contenido, solamente se amplía (y no mucho) aquello que ya conoce.

Los abordajes metodológicos vivenciados por quienes recibieron el contenido en la EF escolar fueron variadas y mostraron cierto equilibrio entre tener la experiencia del contenido y buscar el saber alrededor de este. Sin embargo, este estudio no permitió, por medio de 
cuestionarios, profundizar en el nivel de discusión, de reflexión sobre la práctica y de la problematización que se desarrollaba en las lecciones. Así, ese datos sobre la preocupación conceptual, procedimental y actitudinal se debe interpretar con cautela.

En lo que concierne al abordaje del contenido y sus contribuciones para la vida de los estudiantes, un gran número de los participantes percibe el daño a su conocimiento de forma general y a su capacidad motriz específicamente. Más aún, algunos estudiantes llegan a percibir que esto no los ayuda en el desarrollo de sus cursos de formación profesional y, en especial, con sus posibilidades de aprendizaje en la disciplina de la gimnasia.

Finalmente, se sugiere atender esta realidad en los cursos de formación inicial. Es necesario que los currículum de la disciplina gimnástica, creen estrategias de enseñanzaaprendizaje que promuevan la construcción del conocimiento gimnástico con propiedad y seguridad, atendiendo la falta de experiencia o el desconocimiento de los estudiantes. Esto, para así abrir mayores posibilidades de que los futuros profesores comiencen a cambiar la realidad en su futura práctica profesional. 


\section{REFERENCIAS}

Andrade, T.V.C., Da Silva Rocha, E.C., Paraíso Alves, M. y Vinciprova Fonseca, M.C. (2016). Ligações entre o ensino de ginástica artística escolar e o desenvolvimento motor de crianças: um estudo de revisão. Revista Práxis, 8(16), 123-130. Recuperado de http://revistas.unifoa.edu.br/index.php/praxis/article/view/753

Araújo, T. V. (2017). Educação física e mídia: a relação na escolha de temas e conteúdos. Trabalho de conclusão de curso (Licenciatura). Universidade Federal do Rio Grande do Norte. Recuperado de http://monografias.ufrn.br/handle/123456789/5518

Ayoub, E. (2007). Ginástica geral e educação física escolar (2ª ed.). Brasil: Unicamp.

Barbosa-Rinaldi, I.P. y Cesário, M. (2010). Ginástica Rítmica: da compreensão de sua prática na realidade escolar à busca de possibilidades de intervenção. In: E. Paoliello \& E. Toledo, Possibilidades da ginástica Rítmica (pp. 295-323). São Paulo: Phorte.

Barbosa-Rinaldi, I.P. y Machado de Souza, E.P. (2003). A ginástica no percurso escolar dos ingressantes dos cursos de licenciatura em educação física da universidade estadual de Maringá e da universidade estadual de campinas. Revista Brasileira de Ciência do Esporte, 24(3), 159-173. Recuperado de http://revista.cbce.org.br/index.php/RBCE/article/view/769/0

Bardin, L. (2011). Análise de conteúdo (2ª reimp.). São Paulo: Edições 70.

Barros, I.M.C. (2011). Contributo para a compreensão do processo de (re)construção da identidade professional no contexto da formação inicial: estudo em estudantes estagiários de Educação Física (Dissertação de Mestrado). Universidade do Porto, Portugal. Recuperado de https://repositorioaberto.up.pt/bitstream/10216/57140/2/Teselrenefinal\%204\%20\%20Cpia.pdf

Betti, I.C.R. (1999) Esporte na escola: mas é só isso, professor?, Revista Motriz, 1(1), 25-31. Recuperado de https://bit.ly/2WT2JhU

Betti, M. (1997). A janela de vidro: esporte, televisão e educação física (Tese Doutorado). Universidade Estadual de Campinas. Recuperado de https://bit.ly/36GIXvH

Brasil. (2018). Base Nacional Comum Curricular: Educação é a Base. Brasília: CONSED; UNDIME. Recuperado http://basenacionalcomum.mec.gov.br/images/BNCC El EF 110518 versaofinal site. pdf

Cavalcanti, L.M.B. (2007). Ginástica rítmica na escola: por uma estética do sensível. Paidéia - Revista Brasileira de Ensino de Arte e Educação Física, 2(1) 92-113.

Cervo, A.L. y Bervian, P.A. (1996). Metodologia Científica: para uso dos estudantes universitários ( $4^{\mathrm{a}}$ ed.) São Paulo: Makron Books. 
Daolio, J. (1996). Educação Física Escolar: Em busca da pluralidade. Revista Paulista de Educação Física, (supl.2), 40-42. Recuperado de https://www.revistas.usp.br/rpef/article/view/139646

Graça, A. (2014). A construção da identidade profissional em tempos de incerteza. In: P. Batista, A. Graça \& P. Queirós, O estágio profissional da (re) construção da identidade profissional em educação física (pp. 43-65). Porto: U. PORTO.

Lisbôa, M.M. y Pires, G.L. de. (2016). Quando o esporte-da-mídia vende sonhos e desejos: publicidade e infância na Copa do Mundo da Fifa 2014. In: A.L. Bruggemann, P. Bianchi, S.M. dos Santos, Pesquisa e formação em Mídia-Educação Física (pp.173-186). Florianópolis: Tribo da Ilha. Recuperado de http://docplayer.com.br/55816604-Quandoo-esporte-da-midia-vende-sonhos-e-desejos-publicidade-e-infancia-na-copa-domundo-da-fifa-2014-1.html

Machado de Souza, E.P. (1997). Ginástica Geral: uma área do conhecimento da Educação Física. (Tese Doutorado em Educação Física). Universidade Estadual de Campinas. Recuperado de http://repositorio.unicamp.br/bitstream/REPOSIP/275311/1/Souza ElizabethPaoliello Machadode D.pdf

Maldonado, D.T. y Bocchini, D. (2015). Ensino da ginástica na escola pública: as três dimensões do conteúdo e o desenvolvimento do pensamento crítico. Motrivivência, 27(44),164-176. Recuperado

de https://periodicos.ufsc.br/index.php/motrivivencia/article/view/2175$\underline{8042.2015 \mathrm{v} 27 \mathrm{n} 44 \mathrm{p} 164}$

Marcassa, L. (2004). Metodologia do ensino da ginástica: novos olhares, novas perspectivas. Pensar a Prática, 7(2),171-186. Recuperado de https://revistas.ufg.br/fef/article/view/94/89

Melo Oliveira, G. y de Oliveira Porpino, K. (2010). Ginástica Rítmica e educação física escolar: perspectivas críticas em discussão. Pensar a prática, 13(2),1-18. Recuperado de https://www.revistas.ufg.br/fef/article/view/8632

Merida, F., Nista-Piccolo, V.L. y Merida, M. (2008). Redescobrindo a ginástica acrobática. Movimento, $\quad 14(2), 155-180$.

Recuperado

de https://seer.ufrgs.br/Movimento/article/view/5755

Porto, J. (2014). A ginástica na educação física escolar de estudantes universitários da Universidade Estadual Paulista/Campus de Rio Claro (Licenciatura - Educação Física). Universidade Estadual Paulista, Instituto de Biociências de Rio Claro. Recuperado de https://repositorio.unesp.br/handle/11449/131754?locale-attribute=en

Ramirez Costa, A., de Carvalho Macías, C.C., DA Cunha Faro, C.L. y da Silva Matos, L. (2016). Ginástica na escola: por onde ela anda professor? Conexões: Educação Física, 
Esporte e Saúde, 14(4), 76-96. Recuperado de https://periodicos.sbu.unicamp.br/ojs/index.php/conexoes/article/view/8648071

Dos Santos, S. y Gheller, R.G. (2012). Construção e validação de instrumentos para coleta. In: S. Santos y R. O. Moretti-Pires, Métodos e Técnicas de Pesquisa qualitativa aplicada à Educação Física (pp. 195-206). Florianópolis: Tribo da Ilha.

Schiavon, L. y Nista-Piccolo, V. (2007). A ginástica vai à escola. Movimento,13(3),131-150. Recuperado de https://seer.ufrgs.br/Movimento/article/view/3572

Secretaria de Estado da Educação do Paraná. (2008). Diretrizes Curriculares da Educação Básica. Recuperado de http://www.educadores.diaadia.pr.gov.br/arquivos/File/diretrizes/dce edf.pdf

Soares, C.L., Taffarel, C.N.Z., Varjal, E., Castellani Filho, L., Escobar, M.O. y Bracht, V. (2009). Metodologia do Ensino de Educação Física. São Paulo: Cortez. 